\title{
Antimicrobial resistance in Neisseria gonorrhoeae in China: a meta-analysis
}

\author{
Yawen Chen ${ }^{\dagger}$, Yanhong Gong ${ }^{\dagger}$, Tingting Yang, Xingyue Song, Jing Li, Yong Gan, Xiaoxv Yin ${ }^{*}$ \\ and Zuxun Lu*
}

\begin{abstract}
Background: Neisseria gonorrhoeae (N. gonorrhoeae) resistance to antimicrobial has been a major concern in China, and epidemiological data on N. gonorrhoeae resistance are not well understood. This meta-analysis was aimed at summarizing the evidence on $\mathrm{N}$. gonorrhoeae resistance to penicillin, tetracycline, ciprofloxacin, ceftriaxone and spectinomycin in China.
\end{abstract}

Methods: Two researchers independently searched five databases to identify studies on $\mathrm{N}$. gonorrhoeae resistance to antimicrobials from the databases' inception to November 7, 2014. A random-effects model was used to estimate the antimicrobial resistance rates and their corresponding $95 \%$ confidence intervals (Cls). Publication bias was assessed with the Begg rank correlation test and the Egger test.

Results: We included 127 studies in our synthesis reporting antimicrobial resistance. Our analyses demonstrated that $\mathrm{N}$. gonorrhoeae resistance to penicillin and tetracycline respectively increased from $74.41 \%$ (95 \% Cl: 64.1-84.7 \%) and $68.3 \%$ (95 \% Cl: 58.7-78.0 \%) in 2000 to $84.2 \%$ (95 \% Cl: 79.7-88.8 \%) and 82.4\% (95 \% Cl: 79.9-84.7 \%) in 2012. N. gonorrhoeae resistance to ciprofloxacin experienced a steady increase from $12.7 \%$ (95 \% Cl, 8.6-16.7 \%) in 1995 and reached $93.8 \%$ (95 \% Cl: 91.9-95.7 \%) in 2003. N. gonorrhoeae resistance to ceftriaxone was $1.7 \%$ (95 \% Cl: 0.5-5.7 \%) before 1995 and $0.5 \%$ (95 \% Cl: 0.2-1.4\%) in 2012, and N. gonorrhoeae resistance to spectinomycin was less than $2 \%$ from 1995 to 2012.

Conclusions: $N$. gonorrhoeae resistance rates to penicillin, tetracycline and ciprofloxacin were high in China. Ceftriaxone and spectinomycin remained effective therapy for the treatment of gonorrhea. It is essential to strengthen N. gonorrhoeae resistance surveillance and update treatment guidelines timely.

Keywords: Neisseria gonorrhoeae, Gonorrhea, Antimicrobial, Resistance, Susceptibility

\section{Background}

Neisseria gonorrhoeae ( $N$. gonorrhoeae) is the causative agent of gonorrhea, which is one of the most prevalent sexually transmitted diseases (STD) and causes male urethritis, female endocervicitis, and severe reproductive complications. It also increases the transmission of human immunodeficiency virus, resulting in substantial morbidity and socioeconomic consequences [1]. The World Health Organization (WHO) estimated 106 million new cases of gonorrhea worldwide in 2008 [2, 3]. In

\footnotetext{
* Correspondence: yinxiaoxv@yahoo.com; zuxunlu@yahoo.com ${ }^{\dagger}$ Equal contributors Department of Social Medicine and Health Management, School of Public Health Tongji Medical College, Huazhong University of Science and Technology, Wuhan, Hubei, China
}

China, patients with $N$. gonorrhoeae visited STD clinics or hospital outpatient departments for treatment and 95,263 cases of gonorrhea were reported in 2012, making gonorrhea the sixth most common infectious disease [4] and the first most common STD [5].

Management of $N$. gonorrhoeae infection is a serious challenge in the setting of increasing antimicrobial resistance (AMR) [6-8]. The importance of AMR surveillance has been extensively elucidated $[9,10]$, particularly as treatment options for gonorrhea decreased because of the spread of resistance to sulfonamides, penicillin, tetracycline, and quinolone.

China established a national surveillance program to monitor AMR in 1987. Twenty-five N. gonorrhoeae AMR surveillance sentinel sites were distributed in 25 
provinces of China based on the medical resource distribution and geographical representativeness, which were established by the Chinese Center for Disease Control and Prevention (CDC). N. gonorrhoeae resistance cases were sent to Chinese CDC by sentinel sites. Since 1992, China has been a part of WHO Western Pacific Regional (WPR) Gonococcal Antimicrobial Surveillance Programme. However, we found that only two studies reported $N$. gonorrhoeae resistance to antimicrobials based on nationwide data from the surveillance program [11, 12]. Whereas, more than a hundred reports on N. gonorrhoeae AMR were based on data from one or a few surveillance sentinel sites, and these studies had obvious differences in settings, methods, findings, and some other characteristics. We conducted this systematic review in order to provide a comprehensive understanding of $N$. gonorrhoeae resistance to antmicrobials in China.

\section{Methods}

This meta-analysis was conducted according with the checklist of the Meta-Analysis of Observational Studies in Epidemiology Guideline [13].

\section{Search strategy}

Two independent researchers (Y.W.C and T.T.Y) searched four Chinese biomedical databases (China Biology Medicine disc [CBMdisc] (1978-), China National Knowledge Infrastructure [CNKI] (1915-), VIP Information/Chinese Scientific Journals database (1989-) and Wanfang database (1998-)) and PubMed (1966-) to identify relevant studies from their respectively inception to November 7, 2014. Search terms (in Chinese) included "Neisseria gonorrhoeae" or "gonorrhea", and their combinations with "resistant" or "resistance" or "susceptible" or "susceptibility" in Chinese databases. We used the string '(Neisseria gonorrhoeae or gonococc* or gonorrh*) AND (resistan* or sensitiv* or susceptib*) AND (China or Chinese)' in PubMed. In addition, relevant references from each study were retrieved and further added to the analysis.

\section{Inclusion criteria}

The included studies met the following criteria: (1) an original study published in Chinese or English; (2) conducted in mainland China; (3) determined minimum inhibitory concentrations (MICs) by using the agar dilution method; (4) specified the total number of tested $N$. gonorrhoeae isolates; (5) reported the AMR rate in N. gonorrhoeae isolates, or implied it by indicating their MICs, following the criteria by WHO WPR Resistance Surveillance Programme guidelines[14] or Clinical and Laboratory Standards Institute standards; (6) tested more than $30 \mathrm{~N}$. gonorrhoeae isolates in order to guarantee the quality of testing; and (7) isolated $N$. gonorrhoeae strains from adults (age $\geq 18$ years).

\section{Data extraction}

The following data was extracted from each study: name of first author, publication year, study region, number of tested isolates, and resistance rate. Data from the included studies were independently extracted by two authors (X.Y.S and J.L) and any discrepancies were resolved through the introduction of a third reviewer (X.X.Y).

\section{Quality assessment}

The methodological quality of the included studies was assessed with the recommended approach of the WHO [15]. Briefly, we assessed (1) whether the study described the method of identifying $N$. gonorrhoeae isolates;(2) whether the study specified the location where $N$. gonorrhoeae isolates were collected;(3) whether the study specified the collection period of the isolates; (4) whether the study described the population from which $N$. gonorrhoeae isolates were obtained;(5) whether the study included at least 100 tested N. gonorrhoeae isolates;(6) whether the study utilized control strains recommended by WHO in determining MICs with agar dilution method.

The quality assessment scores of the included studies are shown in Additional file 1: Table S1. Each criterion was rated 1 point if a study satisfied the WHO's recommendation. Two independent reviewers assessed the quality of the included studies. We considered studies that scored 5 or higher as "high quality", 3 or 4 as "moderate quality", and 2 or lower as 'low quality'.

\section{Statistical analysis}

$N$. gonorrhoeae resistance rates to antimicrobials with their corresponding $95 \%$ confidence intervals (CIs) were calculated using the random-effects model. Where a study reported results for years separately, these were regarded as separate reports. Subgroup analyses were carried out by year. If the number of reports in 1 year was less than 3 , these reports were subsumed in the next year. For example, only one report about $N$. gonorrhoeae resistance to tetracycline in 2002; thus, we subsumed this report in 2003 to conduct a meta-analysis. In our study, considering that reports published before 1995 and after 2012 were scarce and the number of reports was less than 3 in any year before 1995 and after 2012, we have subsumed the reports published before 1995 and after 2012 in one subgroup to make our results more understandable.

Statistical heterogeneity among studies was evaluated by using the $I^{2}$ statistic, where values of 25, 50 and $75 \%$ represent cut-off points for low, moderate and high degrees of heterogeneity, respectively [16]. Publication bias was assessed by using the Begg test [17] and the Egger test [18]. All statistical analyses were performed by using Comprehensive Meta Analysis V3. All tests were twosided with a significance level of 0.05 . 


\section{Results}

Literature search

We present the study selection process in Fig. 1. The search identified 5,607 unique articles from PubMed, CNKI, CBMdisc, VIP, and WanFang, of which 270 were identified as potentially relevant. After retrieving the full text for further evaluation, 127 studies that reported $N$. gonorrhoeae AMR rates were included.

\section{Study characteristics}

The main study characteristics and quality assessment results are shown in Additional file 1: Table S1. Of the127 studies (Additional file 2: Table S2), 12 were in English and 115 were in Chinese. These studies were published between 1991 and 2014. In these studies, 42,509 N. gonorrhoeae isolates were examined for their resistance to one or more antimicrobials used for the treatment of gonorrhea. Of the 127 studies, 90 were deemed high-quality studies; 34, moderate-quality studies; and 3, low-quality studies. The $N$. gonorrhoeae resistance rates to penicillin, tetracycline, ciprofloxacin, ceftriaxone and spectinomycin were estimated in our study.

\section{N. gonorrhoeae resistance to antimicrobials}

In 91 studies, 154 reports were found on $N$. gonorrhoeae resistance to penicillin. The pooled $N$. gonorrhoeae resistance rate to penicillin was $58.1 \%$ (95 \% CI: 50.3-65.8\%) before
1995 and increased up to $84.2 \%$ (95 \% CI: 79.7-88.8 \%) in 2012 (Fig. 2).

According to 73 reports, the $N$. gonorrhoeae resistance rate to tetracycline fluctuated over time. The resistance rate was $68.3 \%$ (95\% CI: 58.7-78.0 \%) before 2003 and picked in 2008 (91.7 \%, 95 \% CI: 86.4-97.0 \%; Fig. 2). Between 1994 and 2013, 221 reports from 113 studies were about $N$. gonorrhoeae resistance to ciprofloxacin. The $N$. gonorrhoeae resistance rate to ciprofloxacin reached 93.8 \% (95 \% CI: 91.9-95.7\%) in 2003 and fluctuated between $92.1 \%$ (95 \% CI: 90.1-94.0\%) and 99.3\% (95\% CI: 98.9-99.7 \%) between 2004 and 2012 (Fig. 2).

In 103 studies, 205 reports estimated $N$. gonorrhoeae resistance to ceftriaxone. The pooled resistance rate was 1.7 \% (95 \% CI: 0.5-5.7 \%) before 1995 and $0.5 \%$ (95 \% CI: $0.2-1.4 \%$ ) in 2012 (Fig. 2). N. gonorrhoeae resistance to spectinomycin was reported in 113 studies, with 33,485 N. gonorrhoeae isolates. The pooled $N$. gonorrhoeae resistance rate to spectinomycin was $0.3 \%$ (95\% CI: 0.2-0.8 \%; Fig. 2). Data on $N$. gonorrhoeae resistance to antimicrobials are shown in Additional file 3: Table S3.

\section{Publication bias}

Evidence of high heterogeneity was observed for each antimicrobial agent in our study $\left(I^{2}\right.$ ranged from 64.89 to $95.80 \%, P=0.001)$. The Begg rank correlation test and Egger linear regression test indicated evidence of

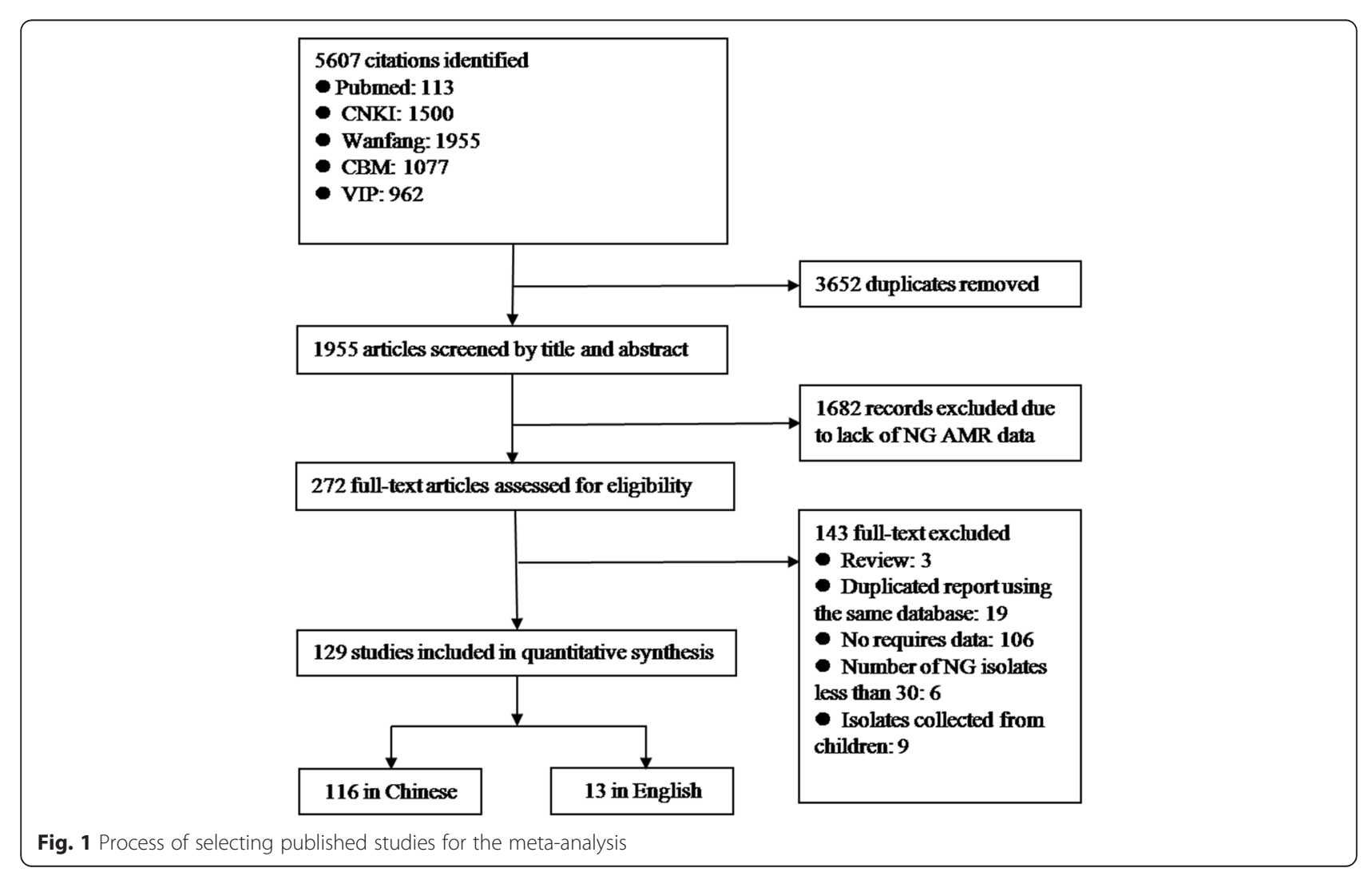




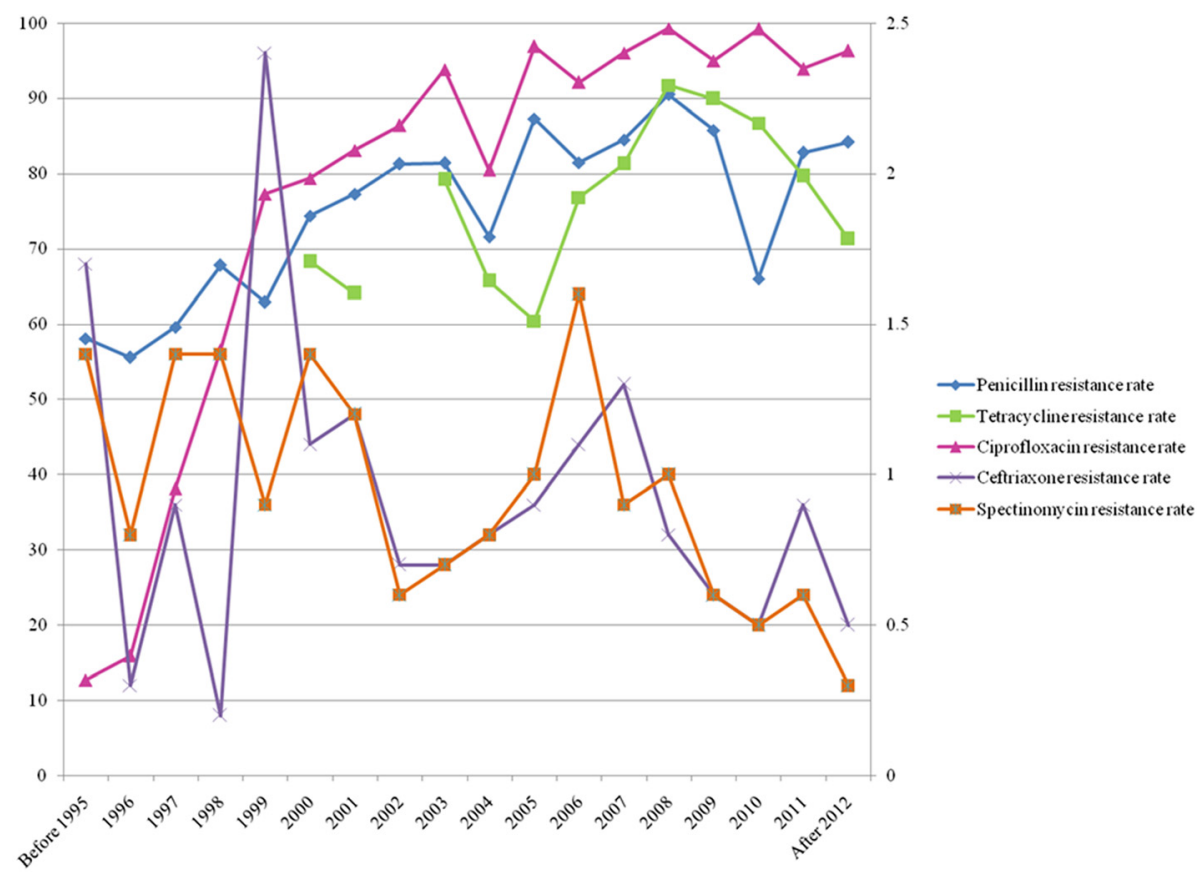

Theright vertical was the ceftriaxone and spectinomycin resistance rates; the left vertial axis was the penicillin, tetracycline, and ciprofloxacin resistance rates.

Fig. 2 N. gonorrhoeae resistance to penicillin, tetracycline, ciprofloxacin, ceftriaxone and spectinomycin, 1995-2012

publication bias among studies for each antimicrobial agent $(P<0.05$ for both tests); Additional file 4: Table S4).

\section{Discussion}

Based on data from 127 studies, including 42,509 tested $N$. gonorrhoeae isolates, $N$. gonorrhoeae resistance to the previously recommended first-line antimicrobials penicillin, tetracycline and ciprofloxacin reached as high as 84.2, 71.3, and $96.3 \%$, respectively, in China. The $N$. gonorrhoeae resistance rates to penicillin and tetracycline were higher in China than in other Asian countries such as Vietnam [9], India, Pakistan and Bhutan [19] during the same period. Ciprofloxacin-resistant $N$. gonorrhoeae isolates in China were much higher than those reported in Laos [20-22] and Vietnam [20-26], and were comparable with those in Thailand $[9,27,28]$. N. gonorrhoeae resistance rate to spectinomycin remains very low, which is in accordance with WHO report [29] and previous studies from other countries in the Western Pacific Region [30-33].

The WHO recommends that routine use of an antimicrobial for treatment should be discontinued when therapeutic failure and/or AMR rate reaches a level of $5 \%$ [34]. The unremitting development of resistance to multiple antimicrobials in $N$. gonorrhoeae isolates has motivated ongoing modification of treatment guidelines in China. Penicillin was recommended as the first choice of treatment of N. gonorrhoeae infections in 1989. By 1995, the $N$. gonorrhoeae resistance rate to penicillin was nearly $60 \%$. The recommended treatment of gonorrhea was then changed to ciprofloxacin in 1995. Afterwards, the $N$. gonorrhoeae resistance rate to ciprofloxacin increased sharply and reached $79.4 \%$ by 2000 . Many studies suggest that the widespread use of antimicrobials was an important cause in the development of AMR [35-38]. We hypothesize that $N$. gonorrhoeae AMR might be contributed to the widespread use of penicillin and ciprofloxacin after they have been recommended for clinical use. In response to extensive $N$. gonorrhoeae resistance to penicillin and ciprofloxacin, ceftriaxone and spectinomycin were recommended as the first-line antimicrobials for the treatment of gonorrhea in 2000 in China. Fortunately, according to our data, $N$. gonorrhoeae resistance to both ceftriaxone and spectinomycin is not widespread. The $N$. gonorrhoeae resistance rates to ceftriaxone and spectinomycin were 0.5 and $0.3 \%$, respectively, in 2012 .

The main reason for the rapid increase of $N$. gonorrhoeae resistance rates to penicillin and ciprofloxacin after they have been recommended for clinical use is likely their indiscriminate use. Gonorrhea was considered to be caused by the sexual misconduct in China in the 1990s; and because of stigma, patients trended to administer antimicrobial treatment by themselves or visited non-formal medical institutions such as unregistered private clinics. Moreover, some patients did not complete the full treatment course, increasing the chance for AMR to develop. In addition, at 
the time, antimicrobial management policies and effective supervision over physicians' prescribing behaviors were lacking in China. For example, patients could buy antimicrobials in drug stores without a prescription. Indeed, an association between self-prescribed antimicrobial use and gonococcal AMR was observed in Philippines [39].

Recognizing the rapid spread and serious consequences of AMR [40], the Ministry of Health of China promulgated "The Guidelines on the Clinical Application of Antimicrobials" in 2004 and "The Guidelines on Prescription Management" in 2006. The Ministry also issued "Prescription Management and Evaluation Standards in Clinical Practice" in 2010. For STDs, the Chinese CDC formulated "The Guidelines for Diagnosis and Treatment of Sexually Transmitted Disease" in 2006; the Ministry of Health modified and promulgated "Prevention and Management of Sexually Transmitted Disease" in 2012, which defined legal responsibility for relevant departments and personnel, and explicitly stated that medical institutions should further standardize the treatment of gonorrhea. In addition, the government strengthened health education on the importance of timely and standardized treatment of gonorrhea. These measures may have potentially contributed to the low resistance rates to ceftriaxone and spectinomycin. However, given that treatment failures with ceftriaxone have been reported not only in China [41-43] but also in Japan, France, and Spain [44-46], and spectinomycin-resistant $N$. gonorrhoeae strains have been detected in China [47-51] and other countries [52, 26], clinicians should closely monitor for the resistance status of patients treated with ceftriaxone and spectinomycin.

Our study has a number of limitations: (1) the included studies were conducted in various regions, mostly with variable sample sizes, which introduced substantial heterogeneity to the data obtained; (2) all tested $N$. gonorrhoeae isolates were collected from patients who attended STD clinics or hospital outpatient departments, which may introduce sample bias for the patients may have a higher prevalence of $N$. gonorrhoeae AMR than the general population; and (3) demographic information such as sex and age and information regarding "risk patient groups" or "risk factors" were lacking.

\section{Conclusions}

$N$. gonorrhoeae resistance rates to penicillin, tetracycline and ciprofloxacin were high in China, while the resistance rates to ceftriaxone and spectinomycin remained lower than $5 \%$. Therefore, ceftriaxone and spectinomycin can be recommended as effective therapy for gonorrhea in China. Nevertheless, considering the emergency of ceftrixone and spectinomycin resistant strains in various regions, it is essential to strengthen $N$. gonorrhoeae resistance surveillance and update treatment guidelines timely based on new evidence as antimicrobial resistance develops.

\section{Ethics approval and consent to particapate}

Not applicable.

\section{Consent for publication}

Not applicable.

\section{Availability of data materials}

The datasets supporting the conclusions of this article are included within the article and its additional files.

\section{Additional files}

Additional file 1: Table S1. Characteristics and quality assessment of studies included in the systematic review. (DOCX 48 kb)

Additional file 2: Table S2. The included studies. (DOCX $23 \mathrm{~kb}$ ) Additional file 3: Table S3. Antimicrobial resistance rate of Neisseria Gonorrhoeae isolates. (DOCX 24 kb)

Additional file 4: Table S4. The Begg and Egger test of heterogeneity. (DOCX $14 \mathrm{~kb}$ )

Abbreviations

AMR: antimicrobial resistance; CBMdisc: China Biology Medicine disc; $\mathrm{Cl}$ : confidence interval; CNKI: China National Knowledge Infrastructure; Neisseria gonorrhoeae: N. gonorrhoeae; STD: sexually transmitted disease; WHO: World Health Organization.

\section{Competing interests}

We declare we have no conflict of interest. No funding was received for this systematic review.

\section{Author's contributions}

YWC, YHG, XXY and ZXL conceived the idea and prepared a draft review protocol. YWC and TTY searched articles. XYS and JL carried out data collection. YWC, YHG, TTY and YG performed the statistical analyses. YWC, $Y H G, Y G$ and $X X Y$ drafted the manuscript. $X X Y$ and $Z X L$ are guarantors of the paper. All authors have reviewed the study findings and read and approved the final version.

Acknowledgements

We thank all the authors of the studies included in our meta-analysis.

Funding

None

Received: 27 August 2015 Accepted: 16 February 2016

Published online: 03 March 2016

References

1. Cohen MS. Classical sexually transmitted diseases drive the spread of HIV-1: back to the future. J Infect Dis. 2012;206(1):1-2.

2. CDC C. Esitimation of Four Sexually Transmitted Diseases Among Adults Aged 15-49 in Global in. 2008. http://www.ncstdc.org/show.asp?id=1217.

3. WHO: Prevalence and incidence of selected sexually transmitted infections: Chlamydia trachomatis, Neisseria gonorrhoeae, syphilis and Trichomonas vaginalis. http://apps.who.int/iris/bitstream/10665/44735/ 1/9789241502450_eng.pdf.

4. CDC C. Reports From National STI Surveillance System in China. http://www ncstdc.org/upfiles/201303/20130319152506703.pdf.

5. Zhang G, Su M, Zhu H, Gao Y. Analysis on human papilloma virus genotype in sex workers with condyloma acuminatum in Kunming. Int J Lab Med. 2015;02:211-2. 
6. Gerbase AC, Rowley JT, Heymann DH, Berkley SF, Piot P. Global prevalence and incidence estimates of selected curable STDs. Sex Transm Infect. 1998;74 Suppl 1:S12-6.

7. Johnson SR, Morse SA. Antibiotic resistance in Neisseria gonorrhoeae: genetics and mechanisms of resistance. Sex Transm Dis. 1988;15(4):217-24.

8. Moodley P, Pillay C, Goga R, Kharsany AB, Sturm AW. Evolution in the trends of antimicrobial resistance in Neisseria gonorrhoeae isolated in Durban over a 5 year period: impact of the introduction of syndromic management. J Antimicrob Chemother. 2001;48(6):853-9.

9. Olsen B, Pham TL, Golparian D, Johansson E, Tran HK, Unemo M. Antimicrobial susceptibility and genetic characteristics of Neisseria gonorrhoeae isolates from Vietnam, 2011. BMC Infect Dis. 2013;13:40.

10. Lebedzeu F, Golparian D, Titov L, Pankratava N, Glazkova S, Shimanskaya I, et al. Antimicrobial susceptibility/resistance and NG-MAST characterisation of Neisseria gonorrhoeae in Belarus, Eastern Europe, 2010-2013. BMC Infect Dis. 2015;15:29.

11. Ye SZ. Survey on antibiotic sensitivity of Neisseria gonorrhoeae strains isolated in China, 1987-1992. Sex Transm Dis. 1994;21(4):237-40.

12. Ye S, Su X, Wang Q, Yin Y, Dai X, Sun H. Surveillance of antibiotic resistance of Neisseria gonorrhoeae isolates in China, 1993-1998. Sex Transm Dis. 2002:29(4):242-5

13. Stroup DF, Berlin JA, Morton SC, Olkin I, Williamson GD, Rennie D, et al. Meta-analysis of observational studies in epidemiology: a proposal for reporting. Meta-analysis Of Observational Studies in Epidemiology (MOOSE) group. JAMA. 2000;283(15):2008-12.

14. WHO. Sensitivity testing of Neisseria gonorrhoeae: Methodologies for use by participants in the WHO western Pacific regional surveillance programme. In: WHO/WPR Resignal Antimicrobial Surevillance Working Group Meeting Proceedings; 1992

15. WHO. Manual for the Laboratory Identification and Antimicrobial Susceptibility Testing of Bacterial Pathogens of Public Health Importance in the Developing World. 2003.

16. Higgins JP, Thompson SG. Quantifying heterogeneity in a meta-analysis. Stat Med. 2002;21(11):1539-58.

17. Begg CB, Mazumdar M. Operating characteristics of a rank correlation test for publication bias. Biometrics. 1994;50(4):1088-101.

18. Egger M, Davey SG, Schneider M, Minder C. Bias in meta-analysis detected by a simple, graphical test. BMJ. 1997;315(7109):629-34.

19. Sethi S, Golparian D, Bala M, Dorji D, Ibrahim M, Jabeen K, et al. Antimicrobial susceptibility and genetic characteristics of Neisseria gonorrhoeae isolates from India, Pakistan and Bhutan in 2007-2011. BMC Infect Dis. 2013;13:35.

20. Tapsall JW, Limnios EA, Abu BH, Darussalam B, Ping YY, Buadromo EM, et al. Surveillance of antibiotic resistance in Neisseria gonorrhoeae in the WHO Western Pacific and South East Asian regions, 2007-2008. Commun Dis Intell Q Rep. 2010;34(1):1-7.

21. Surveillance of antibiotic resistance in Neisseria gonorrhoeae in the WHO Western Pacific Region, 2002. Commun Dis Intell Q Rep 2003 27(4):488-491.

22. Surveillance of antibiotic resistance in Neisseria gonorrhoeae in the WHO Western Pacific Region, 2001. World Health Organization. Commun Dis Intell Q Rep 2002, 26(4):541-545.

23. Surveillance of antibiotic resistance in Neisseria gonorrhoeae in the WHO Western Pacific Region, 2006. Commun Dis Intell Q Rep 2008, 32(1):48-51.

24. Surveillance of antibiotic resistance in Neisseria gonorrhoeae in the WHO Western Pacific Region, 2005. Commun Dis Intell Q Rep 2006, 30(4):430-433.

25. Surveillance of antibiotic resistance in Neisseria gonorrhoeae in the WHO Western Pacific Region, 2004. Commun Dis Intell Q Rep 2006, 30(1):129-132.

26. Surveillance of antibiotic resistance in Neisseria gonorrhoeae in the WHO Western Pacific Region, 2000. Commun Dis Intell Q Rep 2001, 25(4):274-276

27. Lahra MM. Surveillance of antibiotic resistance in Neisseria gonorrhoeae in the WHO Western Pacific and South East Asian Regions, 2010. Commun Dis Intell Q Rep. 2012;36(1):95-100.

28. Surveillance of antibiotic resistance in Neisseria gonorrhoeae in the WHO Western Pacific and South East Asian Regions, 2009. Commun Dis Intell Q Rep 2011, 35(1):2-7.

29. WHO. Antimicrobial Resistance Global Report on Surveillance 2014. 2014.

30. Shimuta K, Unemo M, Nakayama S, Morita-Ishihara T, Dorin M, Kawahata T, et al. Antimicrobial resistance and molecular typing of Neisseria gonorrhoeae isolates in Kyoto and Osaka, Japan, 2010 to 2012: intensified surveillance after identification of the first strain ( $\mathrm{H041)}$ with high-level ceftriaxone resistance. Antimicrob Agents Chemother. 2013;57(11):5225-32.
31. Lahra MM. Annual report of the Australian Gonococcal surveillance programme, 2011. Commun Dis Intell Q Rep. 2012;36(2):E166-73.

32. Tanaka M, Shimojima M, Saika T, lyoda T, Ikeda F, Kanayama A, et al. Nationwide antimicrobial susceptibility survey of Neisseria gonorrhoeae isolates in Japan. Kansenshogaku Zasshi. 2011;85(4):360-5.

33. Tapsall JW. Implications of current recommendations for third-generation cephalosporin use in the WHO Western Pacific Region following the emergence of multiresistant gonococci. Sex Transm Infect. 2009;85(4):256-8.

34. Tapsall JW, Ndowa F, Lewis DA, Unemo M. Meeting the public health challenge of multidrug- and extensively drug-resistant Neisseria gonorrhoeae. Expert Rev Anti Infect Ther. 2009:7(7):821-34.

35. Arason VA, Gunnlaugsson A, Sigurdsson JA, Erlendsdottir H, Gudmundsson $\mathrm{S}$, Kristinsson KG. Clonal spread of resistant pneumococci despite diminished antimicrobial use. Microb Drug Resist. 2002;8(3):187-92.

36. Melander $\mathrm{E}$, Ekdahl $\mathrm{K}$, Jonsson G, Molstad S. Frequency of penicillin-resistant pneumococci in children is correlated to community utilization of antibiotics. Pediatr Infect Dis J. 2000;19(12):1172-7.

37. Kahlmeter G, Menday P, Cars O. Non-hospital antimicrobial usage and resistance in community-acquired Escherichia coli urinary tract infection. J Antimicrob Chemother. 2003;52(6):1005-10.

38. Malhotra-Kumar S, Lammens C, Coenen S, Van Herck K, Goossens H. Effect of azithromycin and clarithromycin therapy on pharyngeal carriage of macrolide-resistant streptococci in healthy volunteers: a randomised, double-blind, placebo-controlled study. Lancet. 2007;369(9560):482-90.

39. Klausner JDAMMV. Correlates of gonococcal infection and of antimicrobialresistant Neisseria gonorrhoeae among female sex workers, Republic of the Philippines, 1996-1997. J Infect Dis. 1999;3(179):729-33.

40. Cosgrove SE, Carmeli Y. The impact of antimicrobial resistance on health and economic outcomes. Clin Infect Dis. 2003;36(11):1433-7.

41. Zhao X. Analysis of the resistance of Neisseria gonorrhoeae to 6 common antimicrobials in Hebei region. J Pathogen Biol. 2014;7:637-41.

42. Gong $Y$, Yu B, Huang $H$. Research of Neisseria gonorrhoeae to antibiotics resistance in Huizhou region. China Mod Med. 2013:22:167-9.

43. Xie J, Chen H. Surveillance of antimicrobial susceptibilities of 233 Neisseria gonorrhoeae isolates. Med Innov China. 2011;17:121-2.

44. Unemo M, Golparian D, Nicholas R, Ohnishi M, Gallay A, Sednaoui P. Highlevel cefixime- and ceftriaxone-resistant Neisseria gonorrhoeae in France: novel penA mosaic allele in a successful international clone causes treatment failure. Antimicrob Agents Chemother. 2012;56(3):1273-80.

45. Ohnishi M, Golparian D, Shimuta K, Saika T, Hoshina S, Iwasaku K, et al. Is Neisseria gonorrhoeae initiating a future era of untreatable gonorrhea?: detailed characterization of the first strain with high-level resistance to ceftriaxone. Antimicrob Agents Chemother. 2011;55(7):3538-45.

46. Camara J, Serra J, Ayats J, Bastida T, Carnicer-Pont D, Andreu A, et al. Molecular characterization of two high-level ceftriaxone-resistant Neisseria gonorrhoeae isolates detected in Catalonia. Spain J Antimicrob Chemother. 2012;67(8):1858-60.

47. Su X, Jiang F, Qimuge, Dai X, Sun H, Ye S. Surveillance of antimicrobial susceptibilities in Neisseria gonorrhoeae in Nanjing, China, 1999-2006. Sex Transm Dis. 2007:34(12):995-9.

48. Qin Y, Zhu D. In vitro susceptibility to 4 antinicrobial agents Neisseria Gonorrhoea. J Chongqing Med Univ. 1991;2:114-7.

49. Gu K, Wu L, Zhu S. Analysis of 81 Neisseria gonorrhoeae isolates resistance to quinolones in Hefei. J Zhejiang Univ. 1992;04:164-6.

50. $\mathrm{Yu}$ K, Yu Q, Mo B. Analysis of Neisseria gonorrhoeae resistance in Nanchang during 2006 to 2008. J Nanchang Univ (Med Sci). 2009; 1:134-135.

51. Zhang L, He L, Wang F. Trend of drug resistance of Neisseria gonorrhoeae in Shenzhen area in 2008. China Trop Med. 2010;5:561-2.

52. Dillon JA, Ruben M, Li H, Borthagaray G, Marquez C, Fiorito S, et al. Challenges in the control of gonorrhea in South America and the Caribbean: monitoring the development of resistance to antibiotics. Sex Transm Dis. 2006;33(2):87-95. 